\title{
Chinese expert consensus statement on the clinical diagnosis and treatment of breast cancer bone metastasis and bone related disease
}

\author{
Zefei Jiang ${ }^{1}$, Haibo Wang ${ }^{2}$, Shu Wang ${ }^{3}$, Shusen Wang ${ }^{4}$, Tao Wang ${ }^{1}$, Xiaojia Wang ${ }^{5}$, Jifeng Feng ${ }^{6}$, \\ Jian Liu', Qiang Liu ${ }^{8}$, Tao Sun', Min Yan ${ }^{10}$, Qingyuan Zhang ${ }^{11}$, Jiayi Chen ${ }^{12}$, Quchang Ouyang ${ }^{13}$, \\ Xichun $\mathrm{Hu}^{14}$, Cuizhi Geng ${ }^{15}$, Peng Yuan ${ }^{16}$, Yongmei Yin ${ }^{17}$, Ning Liao ${ }^{18}$, Yueyin Pan ${ }^{19}$ \\ ${ }^{1}$ Department of Breast Cancer, The Fifth Medical Center of PLA General Hospital, Beijing, China; ${ }^{2}$ Department of Breast surgery, The Affiliated \\ Hospital of Qingdao University, Qingdao, China; ${ }^{3}$ Department of Breast surgery, Peking University People's Hospital, Beijing, China; ${ }^{4}$ Department \\ of Oncology, Sun Yat-sen University Cancer Center, Guangzhou, China; ${ }^{5}$ Department of Breast Cancer, Zhejiang Cancer Hospital, Hangzhou, \\ China; ${ }^{6}$ Department of Oncology, Jiangsu Cancer Hospital, Nanjing, China; ${ }^{7}$ Department of Breast Cancer, Fujian Cancer Hospital, Fuzhou, China; \\ ${ }^{8}$ Department of Breast Cancer, Sun Yat-sen Memorial Hospital, Sun Yat-sen University, Guangzhou, China; ${ }^{9}$ Department of Oncology, Liaoning \\ Cancer Hospital \& Institute, Shenyang, China; ${ }^{10}$ Department of Breast Cancer, Henan Cancer Hospital, Zhengzhou, China; ${ }^{11}$ Department of \\ Oncology, Harbin Medical University Cancer Hospital, Harbin, China; ${ }^{12}$ Department of Radiotherapy, Ruijin Hospital Affiliated to Medical College \\ of Shanghai Jiaotong University, Shanghai, China; ${ }^{13}$ Department of Breast Cancer, Hunan Cancer Hospital, Changsha, China; ${ }^{14}$ Department of \\ Oncology, Fudan University Shanghai Cancer Center, Shanghai, China; ${ }^{15}$ Department of Breast surgery, The Fourth Hospital of Hebei Medical \\ University, Shijiazhuang, China; ${ }^{16}$ Department of Oncology, Cancer Hospital Chinese Academy of Medical Sciences, Beijing, China; ${ }^{17}$ Department of \\ Oncology, Jiangsu Province Hospital, Nanjing, China; ${ }^{18}$ Department of Breast surgery, Guangdong Provincial People's Hospital, Guangzhou, China; \\ ${ }^{19}$ Department of Oncology, Anhui Provincial Hospital, Hefei, China \\ Correspondence to: Zefei Jiang. Department of Breast Cancer, The Fifth Medical Center of PLA General Hospital, Beijing, China. \\ Email: jiangzefei@csco.org.cn.
}

\begin{abstract}
Breast cancer is one of the most common malignancy in Chinese women. Up to $80 \%$ of patients with metastatic breast cancer develop bone metastasis resulting in local bone destruction and skeletal-related events such as pathological fracture and spinal cord compression. Skeletal-related events pose an enormous impact on patients' motor function and quality of life. Therefore, prevention of skeletal-related events is one of the treatment objectives of breast cancer bone metastasis, which often requires participation of multidisciplinary team to optimize therapeutic regimen for individual. Standard anti-tumor therapy includes chemotherapy, endocrine therapy, targeted therapy, immunotherapy, etc. Bone-modifying agents are the basic treatment after the occurrence of bone metastases in addition to anti-tumor therapy. As the disease progresses, patients may receive bone radiotherapy and orthopedic surgery. The purpose of this expert's consensus is to standardize diagnosis and treatment of breast cancer bone metastasis and improve the quality of life of patients.
\end{abstract}

Keywords: Breast cancer; bone metastases; bone-modifying agents

Received: 03 December 2020; Accepted: 02 January 2021; Published: 31 January 2021.

doi: $10.21037 /$ tbcr-20-65

View this article at: http://dx.doi.org/10.21037/tbcr-20-65

\section{Introduction}

Bone metastasis is the most common type of breast cancer metastasis. It occurs in nearly $80 \%$ (about $65-75 \%$ ) of patients with advanced breast cancer and is detected as the first manifestation in $27-50 \%$ of advanced breast cancer patients. The common complications of bone metastases are pain, hypercalcemia, fracture, and spinal cord compression, which can seriously affect the patient's quality of life, and 
may even lead to death. Skeletal-relate-events (SREs) are generally used to assess the efficacy of bone-modifying agents in clinical studies of bone metastasis in breast cancer. The "Clinical Trial Endpoints for the Approval of Cancer Drugs and Biologics Guidance for Industry", issued by the Food and Drug Administration (FDA) in December 2018, defined these SREs. There are four types of SREs: pathological fractures, radiation therapy to the bone, surgery to the bone, and spinal cord compression (1). These are all are major factors that influence the autonomic activities and patients' quality of life (2-4). SREs are usually used as the endpoint of clinical studies of bone-modifying agents. However, in real-world clinical practice, the occurrence of SREs is not an indication for stopping the administration of bone-modifying agents.

The diagnosis and treatment of bone metastasis from breast cancer involve physicians from various departments, including oncologists, radiologists, pathologists, and orthopedists. Therefore, multiple disciplinary team discussions and suggestions are important for deciding upon the optimal diagnosis and treatment strategies for patients.

\section{Diagnostic methods for bone metastasis}

The most commonly used diagnostic methods for bone metastasis include real-time non-invasive imaging and tumor biopsy.

Emission computed tomography (ECT) of bone is the most commonly used imaging tool for the initial screening of bone metastases. This method has the advantages of high sensitivity and whole-body imaging. However, it has some disadvantages, such as low specificity, no indication of osteogenic or osteolytic lesions, and no indication of bone destruction from breast cancer bone metastases. ECT of the bone is recommended as the routine initial screening for breast cancer patients with the symptoms of suspected bone metastases, such as bone pain, pathological fracture, alkaline phosphatase (ALP) elevation, or hypercalcemia. In addition, this method could also be used for the routine examination of patients with early-stage breast cancer with high risk of recurrence, locally advanced breast cancer (LABC), and recurrent metastatic breast cancer.

$\mathrm{X}$-ray imaging, CT scanning, and magnetic resonance imaging (MRI) of bone are the major imaging methods for diagnosing bone metastases. For patients with abnormal results on bone ECT scan, subsequent X-ray imaging, CT scanning, or MRI examination of the bone should be conducted, focusing on the areas suspicious of bone metastases so as to clarify bone destruction and assess the stability of the spine.

Plain X-ray imaging is one of the basic methods for the diagnosis of bone metastasis. It is intuitive and has a high specificity; however, it also has a relatively low sensitivity. CT scanning of bone is the most important imaging method for diagnosing bone metastases. It has high sensitivity and specificity, especially for detecting cortical bone damage. It is also very useful for distinguishing osteogenic from osteolytic lesions. X-ray imaging and CT scanning could be used for the evaluation of efficacy in treating bone metastases.

MRI has high sensitivity in diagnosing bone metastases and can clarify the invasion range of the lesions. However, the specificity of MRI is lower than that of CT. Still, spinal MRI examination is very important for assessing spinal cord compression and stability, as well as identifying the indications for the surgery and radiotherapy of bone metastases. Nonetheless, MRI's unique imaging principles could lead to false positives in the diagnosis; thus, abnormal MRI findings alone cannot confirm the presence of bone metastases or evaluate the efficacies of drug therapy on bone metastases.

Positron emission computed tomography (PET/ CT) is a powerful imaging tool with high sensitivity and specificity for detecting abnormal signals of bone metastases at the early clinical stage. Previous clinical studies have demonstrated that fluorodeoxyglucose (FDG) PET has comparable sensitivity and higher specificity than ECT. Moreover, the value in the follow-up evaluation of disease conditions after the treatment of bone metastases from breast cancer is higher than that of ECT. However, this method is not as intuitive as X-ray imaging or CT scanning of bone; thus, it is not routinely recommended in clinical work.

The detection of metastatic cancer cells in bone biopsy is the gold standard for diagnosing bone metastases from breast cancer. For clinically suspected metastatic bone lesions, especially single-bone lesions in patients without soft tissue or visceral metastases, or bone lesions for which the clinical conditions cannot be clearly assessed, a bone biopsy should be considered to clarify the pathological diagnosis.

Furthermore, biochemical markers that suggest active bone turnover may indicate the possibility of bone destruction, and the necessary imaging examinations are required. Biochemical markers of bone turnover could also be used for the dynamic monitoring of the treatment 
of bone metastases. However, bone metastases cannot be diagnosed solely based on the increase of biochemical markers.

In summary, for the clinical diagnosis of bone metastases from breast cancer, ECT can be used for initial screening; X-ray imaging and CT scanning can clarify the existence of bone destruction; while MRI can clearly display the invasion areas of the metastases, thus helping to assess the spinal stability and the influences of bone metastases on surrounding tissues. The advantages of PET/CT scanning in comparison to the above-mentioned methods require further investigations. All kinds of diagnostic methods should be applied reasonably in clinic, and bone biopsy should be used for pathological diagnosis when it is necessary. The imaging examinations for bone metastases from breast cancer are the major methods for assessing the efficacy of bone metastasis treatment. In clinical practices, the efficacies of treatment for bone metastasis can be assessed according to the symptoms of patients, as well as the imaging manifestations and biochemical markers of bone turnover.

\section{Clinical manifestations of bone metastasis}

Multiple osteolytic lesions are common in bone metastases of breast cancer. However, some osteolytic lesions are misdiagnosed as osteogenic lesions due to excessive calcification after treatment. It is thus necessary to determine whether or not there are osteolytic lesions in the imaging (X-ray or CT) of these patients at the initial diagnosis.

The process of osteolytic bone metastasis is a "vicious circle". The process is initiated by metastatic tumor cells that promote the overexpression of receptor activator of NF- $\kappa$ B ligand (RANKL) by osteoblasts, increasing the differentiation and activation of osteoclasts, thus accelerating bone destruction. The acceleration of osteolysis can promote the release and activation of growth factors, which are stored in bone masses and promote tumor growth. This destructive feedback loop not only accelerates the processes of bone destruction but also promotes the further progression of bone metastases (5).

Generally, bone metastases do not endanger the lives of patients unless accompanied by pathological bone fracture. Yet, severe pain induced by bone metastases can severely influence the quality of life. Patients without visceral metastases generally have a relatively long survival time if the bone metastases are well managed (6).

\section{Treatment of bone metastasis}

\section{Treatment objectives}

The major treatment objectives for breast cancer bone metastases are to (I) prevent and treat SREs, (II) alleviate pain, (III) restore functions and improve quality of life, and (IV) manage tumor progression and prolong survival.

\section{Treatment procedures}

Breast cancer bone metastasis is a systemic disease. The most common treatment methods include chemotherapy, endocrine therapy, molecular targeted therapy, and immunotherapy. Treatments for metastatic bone lesions include bone-modifying agents, surgery, radiotherapy, and analgesics. Physicians should design an individualized comprehensive treatment regimen according to the specific condition of patients (Figure 1).

\section{Treatment principles}

As a recurrent metastatic disease, breast cancer bone metastases should be mainly treated with systemic therapy. Antitumor therapies, including chemotherapy, endocrine therapy, and targeted molecular therapy, could be applied according to the principle of classification-based treatment. Bone-modifying agents, such as denosumab and bisphosphonates (BPs), can prevent SREs and have become the basic treatment drugs for bone metastasis of breast cancer. Appropriate local treatment could help in controlling the symptoms of bone metastases. Orthopedic surgery is commonly used to treat pathological fractures caused by single lesion bone metastases and spinal cord compression caused by bone metastases. Radiotherapy is an effective local treatment to control the progress of bone metastases and relieve pain.

When selecting systemic treatment regimens, hormone receptors [estrogen receptor/progesterone receptor (ER/PR)] status, human epidermal growth factor receptor 2 (HER-2) status, age, menstrual status, and disease progression speed need to be considered. In general, endocrine therapy is the preferred treatment option for hormone-responsive breast cancer patients with slow disease progression. Monochemotherapy is the first recommendation for patients with negative hormone receptors. Chemotherapy is the priority for recurrent metastatic breast cancer patients with fast disease progression, especially critical patients with visceral metastases. The strategy of combined or 


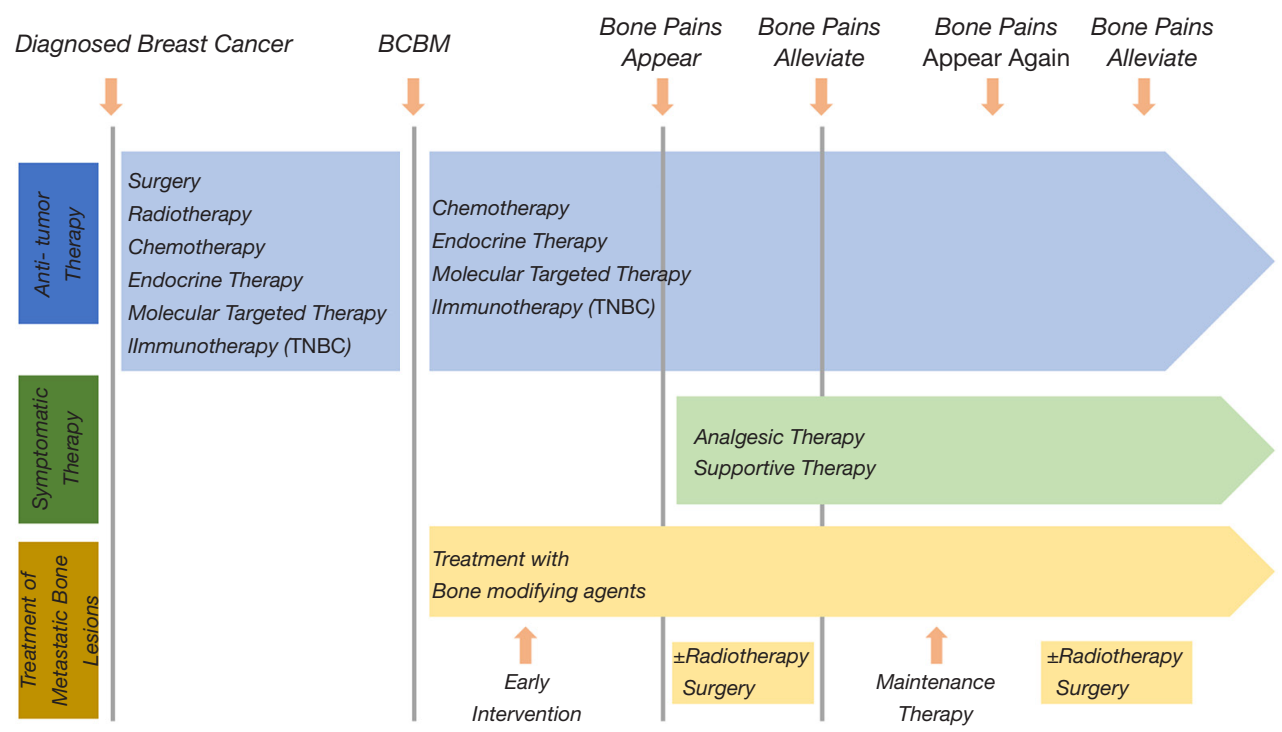

Figure 1 Comprehensive treatment procedure for bone metastases from breast cancer.

monochemotherapy is decided upon according to tumor burden. Regimens containing combined anti-HER-2 agents should be considered in the treatment of patients with HER-2 overexpression (7).

The characteristics of slow-progressive recurrent metastatic breast cancer are the following: (I) the primary and/or recurrent metastatic tumor tissues are ER and/or PR positive; (II) the postoperative disease-free survival (DFS) is over 5 years; (III) the patients only has soft tissue and bone metastases, or asymptomatic visceral metastases.

Regarding hormone-responsive breast cancer, patients with one or more of the following features could possibly benefit from endocrine therapy: (I) ER and/or PR positive in primary and/or recurrent metastatic tumor tissues; (II) elderly patients; (III) long postoperative DFS; (IV) benefit derived from previous endocrine therapy.

\section{Endocrine therapy, chemotherapy, and HER-2-targeted therapy}

\section{Endocrine therapy}

Because breast cancer bone metastasis does not directly pose a threat to life, patients without visceral metastasis have a relatively long survival. Endocrine therapy is preferred for hormone receptor-positive patients. For patients with advanced breast cancer, endocrine therapy's clinical benefit is considerable if the disease conditions are stabilized. The survival time of patients with disease conditions stable for over 6 months is equal to patients with clinical remissions [complete response + partial response $(\mathrm{CR}+\mathrm{PR})]$.

The basic principles of the endocrine therapy for bone metastasis of postmenopausal breast cancer with positive hormone receptor are the following: aromatase inhibitors (AI)+CDK4/6 inhibitors, including monotherapy by fulvestrant or AI, or tamoxifen (TAM), are preferred for endocrine therapy-naïve patients; $\mathrm{AI} /+\mathrm{CDK} 4 / 6$ inhibitors or $\mathrm{AI}+$ histone deacetylase (HDAC) inhibitors are preferred for patients with TAM treatment failure, for whom fulvestrant + CDK4/6 inhibitors, along with monotherapy by fulvestrant or AI, could also be used; fulvestrant + CDK4/6 inhibitors or steroidal AI + HDAC inhibitors are preferred for patients with non-steroidal AI treatment failure, for whom steroidal AI + CDK4/6 inhibitors, along with monotherapy by fulvestrant or steroidal AI, could also be used; fulvestrant + CDK4/6 inhibitors are preferred for patients with steroidal AI treatment failure, while monotherapy by fulvestrant, along with non-steroidal AI + CDK4/6 inhibitors could also be used.

Chemotherapy could also be used for premenopausal patients. Compared to chemotherapy, endocrine therapy is more suitable for long-term treatment. Once the patient receives benefit, the quality of the patient's life improves when a long disease remission time is achieved following the treatment. Therefore, the treatment duration could be prolonged to help achieve long-term management of the disease. In addition, strategies for postmenopausal patients 
could also be applied for premenopausal patients after the suppression of ovarian function.

\section{Chemotherapy}

Chemotherapy should be considered for breast cancer bone metastasis patients with both negative ER and PR, with short postoperative disease free interval, those with rapid progression of the disease accompanied by visceral metastases, or those who did not respond well to endocrine therapy. The most common chemotherapeutic drugs include anthracycline $(\mathrm{A})$, taxanes $(\mathrm{T})$, capecitabine $(\mathrm{X})$, vinorelbine, gemcitabine $(\mathrm{G})$, and eribulin.

A TA regimen (anthracycline accompanied by taxanes) could be selected for patients who were not previously treated with anthracycline or taxanes (adjuvant therapy). For patients who did not respond well to anthracycline adjuvant therapy, XT (capecitabine accompanied with docetaxel) and GT (gemcitabine accompanied with paclitaxel) regimens could be used. For patients who did not respond well to paclitaxel treatment, capecitabine, vinorelbine, gemcitabine, and platinum are recommended. Monochemotherapy is preferred for patients simply with bone metastases, while for patients with visceral metastases and those with extensive bone metastases accompanied by pain, combined chemotherapy is recommended. After the effects of combined chemotherapy are confirmed, these patients should switch to monotherapy for maintenance treatment.

\section{HER-2-targeted therapy}

For patients who were not previously treated with trastuzumab $(\mathrm{H})$ or used it but are still eligible to use it again, THP (docetaxel, trastuzumab, and pertuzumab) double-targeted therapy or TH-based treatment regimens are preferred. Pyrotinib accompanied by capecitabine is recommended for those who do not respond well to $\mathrm{H}$ treatment. Trastuzumab emtansine (T-DM1) treatment, as well as capecitabine combined other tyrosine kinase inhibitors (TKI) could also be selected for these patients.

\section{Radiotherapy}

Radiotherapy is an effective palliative therapy for breast cancer patients with bone metastases. The goal of radiotherapy is to prevent or alleviate symptoms or dysfunctions induced by metastatic bone lesions. Bone pain is a common symptom of bone metastases and one of the major causes influencing patient's life quality and mobility.
The risk of metastasis in weight-bearing bones, such as the spine and femur, accompanied by a pathological fracture, is about $30 \%$. Pathological fracture substantially influences the quality of life and survival of patients. The effects of radiotherapy in treating breast cancer bone metastases include effectively alleviating bone pain and reducing pathological fracture risk. Moreover, the combination of radiotherapy with molecular subtype-specific antitumor drugs and bone-modifying agents could improve the effectiveness of the treatment.

External irradiation on local metastatic bone lesions by a high-energy radiative probe is commonly recommended for breast cancer patients with bone metastases. Effective external irradiation has been found to achieve symptom remission in about $50-80 \%$ cases; among these, one-third of patients could achieve complete remission and significant pain alleviation. The major indications for external irradiation are as follows: (I) for patients with symptomatic bone metastases, the external irradiation could be used to alleviate bone and help to restore functions; (II) it can be selectively used for patients without evident pain and with weight-bearing bones metastases, such as metastasis of the spine or femur.

The commonly used doses and segmentations of external irradiation are $40 \mathrm{~Gy} / 20 \mathrm{~F} / 4 \mathrm{w}, 30 \mathrm{~Gy} / 10 \mathrm{~F} / 2 \mathrm{w}, 20 \mathrm{~Gy} /$ 4 F/2 w, 23 Gy/4 F/3 w, and 8 Gy/F. Previous studies have shown that the symptom remission rate is similar for different dose segmentation regimens. Therefore, longterm radiotherapy with a duration of longer than 2 weeks is not recommended for the palliative radiotherapy of bone metastases unless the metastatic areas are adjacent to vital organs. Thus, relatively low fractionated doses are applied to reduce the late-stage reactions in normal tissues. The medical expenditure of single-dose 8 Gy radiotherapy is substantially lower than that of fractionated irradiation. However, high risk of pathological fracture and repeated symptoms requiring secondary radiotherapy is higher than that of fractionated irradiation. Thus, single-dose 8 Gy radiotherapy is suitable for advanced patients with difficulties in daily activities or movement (8).

Specific highly conformal radiotherapy techniques, such as stereotactic radiotherapy (SBRT), have the advantages of providing rapid dose reduction, thus protecting the vital organs adjacent to the metastatic lesions. These techniques are especially suitable for patients with spinal metastases, particularly those with repeated symptoms requiring secondary treatments. The application of precise radiotherapy has higher requirements for the stability of 
fixed position and rationality of delineating target zones. Thus, it must be applied with caution after strict quality controls have been performed.

Surgical treatment for bone metastases, especially kyphoplasty and vertebroplasty for patients with spinal metastases, could rapidly restore spinal stability. Although surgery is not a contraindication for palliative radiotherapy, there are no sufficient clinical data that can inform a consensus regarding the timing between the combination of these two therapies.

Radionuclide treatment (RNT) is also known as "internal irradiation". It induces antitumor effects by an intravenous injection of isotopic drugs with high affinity to bone, which generates a biological absorption dose within the metastatic bone lesions through the decay of isotopic drugs. RNT has a certain effect on improving the osteolytic lesions and is mainly suitable for patients with a wide distribution of bone metastases, while external irradiation is not able to treat all the lesions with symptoms. RNT has certain effects on relieving pain. Nonetheless, the incidence of bone marrow suppression is relatively high after RNT, and the duration of recovery is also relatively long. Therefore, RNT should only be applied with caution in clinical practice, and suitability of cases and treatment timing need to be sufficiently considered before applying RNT.

Although radiotherapy is the major local treatment method for improving symptomatic bone metastases, the improvement of symptoms cannot occur until the irradiation beam exerts its antitumor effects and the bone recovery reaches a certain degree. Therefore, radiotherapy could be used to replace bone-modifying agents or analgesic agents. For patients with no evident symptom remission or those for whom pain cannot be completely controlled, analgesics should also be provided according to the principles of three-step therapy.

\section{Surgical intervention}

Surgical treatment is generally used to reduce bone metastasis-related complications. The advancement of orthopedic surgery techniques could maximally address the issues of decreased bone strength, pathological fracture, and compression of nerves by tumors in patients with bone metastases, and can further alleviate pain, restore limb function, and consequently improve the quality of life. For patients with bone metastases, close monitoring should be applied in the early detection of metastatic bone lesions. Appropriate judgment should be made regarding the necessity of surgery on long bones with potential pathological fractures. At the same time, effective surgical treatments should be performed before fracture and paraplegia, thus improving patients' quality of life as much as possible.

The surgical treatment methods include the following: simple internal fixation, focal clearance plus internal fixation, focal excision plus artificial joint replacement, decompression for patients with spinal cord compression, and reconstruction of spinal stability. Fixation can be considered as a selective treatment for breast cancer patients with bone metastases who have pathological fracture or decompression due to spinal cord compression, and whose expected survival time is longer than 3 months; for these patients, the anticipated survival is $>3$ months. Prophylactic could be selectively considered for patients with a diameter of femoral metastases $>2.5 \mathrm{~cm}$, or with femoral neck metastases, or cortical bone destruction $>50 \%$; for these patients, the anticipated survival is $>3$ months. The expert group suggests that orthopedists should be consulted to decide upon the appropriate surgery timing (9).

The following factors should be considered when deciding the surgical treatment regimen: (I) sensitivity of the tumor to radiotherapy, chemotherapy, and hormone therapy, in addition to the onset time of the effects; (II) molecular tumor subtypes; (III) risk of pathological fracture, and the presence or risk of spinal cord compression; (IV) spinal stability or pain intensity; (V) anticipated survival of patients; (VI) tolerability to surgery and anesthesia of the systemic conditions (Karnofsky or Burchenal score); (VII) surgical conditions of soft tissues and bones; (VIII) presence of visceral metastases, and the time when metastatic lesions appear.

\section{Treatment with analgesics}

Using analgesics is a very common approach to alleviating pain in cancer patients. The treatment with analgesics for bone metastasis-related pain must abide by the $W H O$ Guidelines of Pain Relief Ladder for Cancer Pains as follows: (I) initial administration of oral or non-invasive drugs; (II) ladder drug administration; (III) timely use of drugs; (IV) individualized drug administration; (V) attention paid to detail (10).

The analgesics include non-steroid anti-inflammatory drugs (NSAIDs), opioids, and adjuvant drugs. The commonly used NSAIDs are acetaminophen, ibuprofen, diclofenac sodium, indomethacin, naproxen, celecoxib, 
and lornoxicam. Controlled-release morphine tablets, fentanyl transdermal patches, controlled-release oxycodone hydrochloride tablets, instant-release morphine tablets, codeine, and methadone are the most commonly used opioids. Pethidine is not suitable for the treatment of cancer pain. Adjuvant drugs include tricyclic antidepressants, anticonvulsants, neuroleptics, and glucocorticoids.

NSAIDs are the basic drugs for the analgesic treatment of bone metastasis pain. When the analgesic effects are suboptimal or moderate-to-severe pain appears, a combination of NSAIDs with opioids is recommended. Timely treatment with controlled-release opioids could favor the continuous alleviation of bone pain. In addition to continuous chronic pain, about $63 \%$ of bone metastasis patients present with pain outbreak. For patients with frequent episodes of pain outbreak, increased and timely dosing of analgesics could help alleviate the pain. However, for patients in whom the increase of analgesic dose produces suboptimal analgesic effects or for those with adverse reactions, the best method is to use quickacting or short-acting analgesics (the single dose is about $5-10 \%$ of the daily dose). For patients with refractory pain outbreaks, patient-controlled pumping can be used for drug administration. For patients with pathological pain, adjuvant drugs should be selected according to the disease conditions. For instance, the combination of tricyclic antidepressants (e.g., amitriptyline, nortriptyline, and doxepin) could be used for patients with burning pain or distending pain. Anticonvulsants such as gabapentin or carbamazepine can be used in combination for patients with electric shock-like pain or gunshot-like pain, and hormones can also be used in combination if necessary. Analgesics can be used in combination with bone-improving agents and radiotherapy for combined therapy.

\section{Principles of clinical application of bone- modifying agents in breast cancer}

\section{Bone-modifying agents}

\section{BPs}

(I) Mechanism of action: BPs are pyrophosphate analogues that inhibit bone resorption by inducing apoptosis of osteoclasts. There is also evidence that BPs can inhibit the proliferation, invasion, and adhesion of tumor cells to bone matrix (11).

(II) Treatment recommendations: the role of BPs is to prevent SREs, reduce cancer treatment-induced bone loss (CTIBL), and increase bone mass density (BMD). Clinical studies have demonstrated that BPs can effectively treat bone metastases from breast cancer. As recommended by the National Institute for Clinical Excellence (NICE) of the UK, BPs are currently used to treat advanced breast cancer complications. Moreover, subsequent clinical studies have demonstrated that BPs could prevent SREs in breast cancer patients with bone metastases. Therefore, for such patients, BPs should be used in addition to chemotherapy and hormone therapy if the expected survival time is more than 3 months and serum creatinine $(\mathrm{Cr})$ is lower than $3.0 \mathrm{mg} / \mathrm{dL}$.

(III) Dosage and administration: the chemical structures of BPs are different from those of the side chains connected by the central carbon atom, and the clinical activities and efficacies of BPs may be different (12).

The first-generation BPs are represented by clodronate disodium. Clodronate disodium can be used intravenously or orally. The oral preparation of clodronate disodium is more convenient for patient-administration at home, or for the combined treatment with endocrine drugs. Meanwhile, intravenous dripping consists of clodronate disodium $400 \mathrm{mg} / \mathrm{d}$, which is given for 3 successive days, followed by oral intake of clodronate disodium $(1,600 \mathrm{mg} / \mathrm{d})$ for 1 cycle (3-4 weeks in total). Clodronate disodium is mainly cleared through the kidneys; thus, sufficient water intake must be maintained during the treatment with clodronate disodium. The clodronate disodium capsules need to be swallowed whole. Under no circumstances should clodronate disodium be used with calcium-containing or other divalent cationcontaining milk, food, or drugs; this may significantly reduce the absorption of clodronate disodium.

The second-generation BPs are nitrogen-containing BPs, such as pamidronate disodium and alendronate sodium. Inhibition of bone resorption in vitro has been shown to be stronger than the first-generation BPs. The dosage and administration of pamidronates includes intravenous dripping of $60-90 \mathrm{mg}$ for injection, an infusion time $\geq 2$ hours, with 1 injection every $3-4$ weeks.

The third-generation BPs are nitrogen-containing BPs with heterocyclic structures (such as zoledronic acid) and nitrogen-containing ibandronates without ring structures. The efficacy of the third-generation BPs is higher than that of the first- and second-generation BPs. The dosage and administration of the third-generation BPs include $4 \mathrm{mg}$ of zoledronic acid intravenously injected for $>15 \mathrm{~min}$, 
Table 1 Recommendations for bone-modifying agent use

\begin{tabular}{lcc}
\hline Expert opinion & $\begin{array}{c}\text { Bone-modifying agents } \\
\text { recommended }\end{array}$ & $\begin{array}{c}\text { Bone-modifying agents not } \\
\text { recommended }\end{array}$ \\
\hline Hypercalcemia of malignancy & $\sqrt{ }$ \\
Bone pain caused by bone metastases, abnormal ECT but normal X-ray, no \\
bone destruction detected by CT or MRI \\
Abnormal ECT, bone metastases confirmed by X-ray (or CT, MRI) \\
$\begin{array}{l}\text { Abnormal ECT, normal X-ray, bone destruction detected by CT or MRI } \\
\text { Imaging diagnosis of bone destruction, regardless of bone pain. }\end{array}$ \\
Abnormal ECT, normal X-ray, and no bone destruction detected by CT or MRI \\
$\begin{array}{l}\text { The risk of bone metastases (lactic dehydrogenase or alkaline phosphatase } \\
\text { elevation), but no imaging evidence of bone metastases }\end{array}$ \\
\hline
\end{tabular}

ECT, emission computed tomography; MRI, magnetic resonance imaging; CT, computed tomography.

at 1 injection every 3-4 weeks; and $6 \mathrm{mg}$ of ibandronate, intravenously injected for $>15 \mathrm{~min}$, at 1 injection every 3-4 weeks. (I) Treating metastatic bone lesions with ibandronates includes a routine dose of $6 \mathrm{mg}$, at 1 injection every 3-4 weeks, with the time of each intravenous injection being $\geq 15 \mathrm{~min}$. (II) The loading dose of ibandronates includes a loading dose of ibandronates that can rapidly alleviate pain in patients with drastic metastatic pain. The method of the drug administration is the following: $6 \mathrm{mg} / \mathrm{d}$ of intravenous injection for 3 continuous days, followed by the routine administration of $6 \mathrm{mg} /$ time every $3-4$ weeks.

\section{Novel bone-modifying agent: denosumab}

(I) Mechanism of action: denosumab is a fully human immunoglobin G2 (IgG2) monoclonal antibody, which can inhibit the formation, function, and survival of osteoclasts via binding to RANKL (receptor activator of NF- $\mathrm{NB}$ ligand). The subtypes of IgG2 monoclonal antibody have little associated effector function (for example, antibody-dependent cell-mediated cytotoxicity or complement-dependent cytotoxicity) (13). Denosumab has no limitations with respect to renal function as it is a monoclonal antibody, which is eliminated by intracellular catabolism in phagocytes in a manner similar to the clearance mechanism of other therapeutic monoclonal antibodies, with no evidence of renal effects.

(II) Treatment recommendations: denosumab is recommended for the (i) prevention of SREs, (ii) the treatment of hypercalcemia, (iii) and the treatment of pain caused by bone metastases. Clinical studies have demonstrated that denosumab could effectively prevent the occurrence of SREs in breast cancer patients. A phase III clinical study in breast cancer patients with bone metastases has shown that denosumab is superior to zoledronic acid in delaying or preventing SREs, as well as improving pain prevention and comparable pain palliation in breast cancer patients with bone metastases. It also has better performance in the voidance of renal toxicity, superior acute phase reactions, and involves a more convenient subcutaneous injection (14-16).

(III) Dosage and administration of denosumab: denosumab should be administered at $120 \mathrm{mg}$ every 4 weeks as a subcutaneous injection in the upper arm, upper thigh, or abdomen.

\section{Recommendations for bone-modifying agents use}

For the recommendations for bone-modifying agents, see Table 1.

\section{Considerations and precautions for bone-modifying agents} use

(I) Serum electrolyte levels, including serum creatinine, serum calcium, phosphates, and magnesium, must be measured before using bone-modifying agents.

(II) Clinical studies have demonstrated that BPs can be used to treat breast cancer bone metastases, hypercalcemia, and bone pain. These agents can also prevent the occurrence of SREs. In addition, studies have shown that the 
third-generation BPs have a better efficacy and safety profile compared to the first and second generation.

(III) The general condition of the patient and disease, as well as the treatment received, should simultaneously be taken into consideration when choosing drug treatment. Intravenous injection of zoledronic acid and ibandronates have shorter infusion time compared to other first and second generations of BPs. Denosumab can be subcutaneously administered; thus, it is very convenient for application in outpatient departments.

(IV) Drug combination of different bone-modifying agents is not recommended. Yet, bone-modifying agents can be used in combination with radiotherapy, chemotherapy, endocrine therapy, and analgesics.

(V) All patients are strongly recommended to take daily calcium and vitamin D supplements: $500 \mathrm{mg}$ calcium and 400 IU vitamin D during denosumab treatment; $1,200-1,500 \mathrm{mg}$ calcium and 400-800 IU vitamin D during the long-term treatment of BPs.

(VI) Dose adjustment of denosumab is not needed in patients with renal impairment. However, close monitoring is needed to avoid hypocalcemia for patients with a creatinine clearance rate $(\mathrm{CCr})$ $<30 \mathrm{~mL} / \mathrm{min}$ or those on dialysis. BPs are excreted through the kidneys. The doses of BPs do not need to be adjusted in patients with mild or moderate renal dysfunctions ( $\mathrm{CCr}>30 \mathrm{~mL} / \mathrm{min}$ ); however, for patients with severe renal dysfunction $(\mathrm{CCr} \leq 30 \mathrm{~mL} / \mathrm{min})$, the risk and benefits of the treatment need to be evaluated beforehand.

(VII) As several previous studies have reported the risk of osteonecrosis of the jaw (ONJ) in a few patients after long-term use of bone-modifying agents, oral examinations need to be performed before using bone-modifying agents. During the medication period, strict oral hygiene and avoidance of oral surgical intervention, including tooth extraction, are recommended. Maxillofacial bone exposure and nonunion during the medication period should be treated by a specialist as soon as possible (17).

\section{Duration and withdrawal of bone-modifying agents}

\section{Duration of treatment}

Previous studies have provided safety data on more than 3 years of treatment with denosumab and more than 2 years of treatment with BPs for metastatic breast cancer.
Therefore, using drugs over 2 years in clinical practice is recommended. However, the treatment duration should be appropriately adjusted according to the safety and clinical benefits.

Different treatment duration for bone-modifying agents is recommended according to treatment objectives: (I) for patients with bone metastases from breast cancer, at least 2 years of treatment is recommended. However, the continuous use should be encouraged when it is safe and effective; (II) to prevent CTIBL of breast cancer patients, 5 years of treatment (every 6 months) is recommended.

If the bone-modifying agent is the only systemic treatment being applied after the discontinuation of chemotherapy, the treatment interval could be increased during the maintenance treatment.

\section{Indications for drug withdrawal}

Indications for drug withdrawal are the following: (I) defined bone-modifying agent-related adverse reactions during treatment; (II) tumor progression during the treatment or metastasis to vital organs; (III) considered necessary by the physicians based on the disease conditions. It should be emphasized that effective bone pain alleviation after other antitumor treatments is not an indicator of treatment discontinuation.

\section{Biochemical markers}

Biochemical markers of bone can reflect bone turnover speed during bone metastasis and suggest the severity of bone destruction and repair. Previous studies have shown that baseline and intratreatment bone biochemical marker levels, such as urinary bone resorption marker crosslinked $\mathrm{N}$-terminal telopeptide of type 1 collagen ( $\mathrm{uNTx}$ ) and osteogenesis marker bone-specific ALP (BAP), are associated with the prognosis of breast cancer patients with bone metastases. Moreover, a meta-regression analysis based on 17 large-scale studies revealed a statistically significant positive relationship between uNTX reduction at week 13 from baseline and SRE risk reduction in patients with bone metastases from solid tumors or bone lesions from multiple myeloma (18). Advanced cancer patients with bone metastases generally have elevated uNTX, while the effect of bone-modifying agents in restoring normal uNTX levels could reduce the risk of SREs. Bone turnover markers could be used as surrogate indicators in clinical studies or important reference indicators to treat breast cancer with bone-modifying agents. Meanwhile, the expert 
consensus does not recommend the routine adjustment of bone-targeting agents in clinical practice according to biochemical changes.

\section{Switching strategy after the occurrence of SREs}

If specific SREs or complications (such as hypercalcemia, bone surgery, and radiotherapy) occur during bonemodifying agent therapy, the drug needs to be discontinued as the endpoint of clinical research. However, it should not be stopped in clinical practice. If SREs occur during the treatment with BPs, denosumab or other BPs could be used instead. A retrospective study has shown that switching to denosumab in patients previously treated with BPs could delay subsequent SREs (19). One clinical trial on treatment with first- and second-generation BPs (clodronates and pamidronates) has shown that switching to zoledronic acid could significantly alleviate pain $(\mathrm{P}<0.001)$ and decrease urine NTX level $(\mathrm{P}=0.008)$ at 8 weeks of treatment, after the occurrence of SREs or bone metastasis progression. However, more clinical data are needed to further investigate whether or not such drug changes could benefit patients.

\section{$C T I B L$}

CTIBL may occur in patients of different ages after chemotherapy and hormone therapy, and may especially involve ovarian function suppression and aromatase inhibitor application. The guidelines of bone health in breast cancer patients issued by the American Society of Clinical Oncology (ASCO) recommended that all breast cancer patients should receive osteoporosis risk evaluation. The high-risk patients include the patients $\geq 65$ years old and patients between 60-64 years old with one or more of the following: (I) a family history of osteoporosis; (II) body weight $<70 \mathrm{~kg}$; (III) previous non-traumatic fracture or risk factors of osteoporosis-induced pathological fracture; (IV) postmenopausal status or history of aromatase inhibitor treatment; $(\mathrm{V})$ premenopausal women on treatments which may induce early menopause (chemotherapy or ovarian suppression).

During adjuvant therapy of breast cancer, bisphosphonate treatment should be initiated if the BMD score (T-Score) is $<-2.5$; bisphosphonate treatment should be considered if the T-score is between -2.5 and -1.0 ; bisphosphonate treatment is not recommended if the T-score is $>-1.0$. Using BPs to treat osteoporosis is different from the methods of treating bone metastases, which could be administered every 3-6 months. Rather, the drug therapy regimen needs to be adjusted according to the BMD score changes after the treatment.

Three large-scale clinical studies, including Z-FAST, ZO-FAST, and E-ZO-FAST (20-22), investigated the effects of zoledronate on preventing bone loss induced by endocrine therapy of breast cancer. The findings showed that early administration of zoledronate in patients who received adjuvant therapy by letrozole could significantly increase the bone density of the lumbar vertebrae and hip. This suggested that injecting $4 \mathrm{mg}$ of zoledronate every 6 months could effectively prevent CTIBL in breast cancer patients on aromatase inhibitor treatment. An ABCSG-12 study reported a case of premenopausal women with breast cancer who were first treated with drug-induced ovarian ablation combined with tamoxifen or anastrozole, and then with zoledronate ( $4 \mathrm{mg}$ per 6 months). The 5 -year follow up showed that zoledronate could effectively prevent treatment-related bone loss (23). The expert consensus suggests that zoledronate could be considered to prevent bone loss induced by endocrine therapy of breast cancer.

Subcutaneous injection of $60 \mathrm{mg}$ denosumab twice per year could effectively prevent CTIBL. The ABCSG-18 study (24) showed that denosumab could reduce the risk of clinical fractures and increase bone density in postmenopausal women with breast cancer receiving aromatase inhibitors.

\section{Acknowledgments}

Funding: None.

\section{Footnote}

Peer Review File: available at http://dx.doi.org/10.21037/ tbcr-20-65

Conflicts of Interest: The authors have completed the ICMJE uniform disclosure form (available at http://dx.doi. org/10.21037/tbcr-20-65). ZJ serves as an Editor-in-Chief of Translational Breast Cancer Research. HW, SW, TW, XW, QL, TS, QZ, JC, CG, YY, PY serve as the unpaid editorial board members of Translational Breast Cancer Research from Mar 2020 to Feb 2022. The other authors have no conflicts of interest to declare.

Ethical Statement: The authors are accountable for all aspects of the work in ensuring that questions related 
to the accuracy or integrity of any part of the work are appropriately investigated and resolved.

Open Access Statement: This is an Open Access article distributed in accordance with the Creative Commons Attribution-NonCommercial-NoDerivs 4.0 International License (CC BY-NC-ND 4.0), which permits the noncommercial replication and distribution of the article with the strict proviso that no changes or edits are made and the original work is properly cited (including links to both the formal publication through the relevant DOI and the license). See: https://creativecommons.org/licenses/by-nc-nd/4.0/.

\section{References}

1. FDA. Clinical Trial Endpoints for the Approval of Cancer Drugs and Biologics Guidance for Industry. Available online: https://www.fda.gov/media/71195/download

2. Coleman RE. Metastatic bone disease: clinical features, pathophysiology and treatment strategies. Cancer Treat Rev 2001;27:165-76.

3. Johnson JR, Williams G, Pazdur R. End points and United States Food and Drug Administration approval of oncology drugs. J Clin Oncol 2003;21:1404-11.

4. Kinnane N. Burden of bone disease. Eur J Oncol Nurs 2007;11 Suppl 2:S28-31.

5. Roodman GD. Mechanisms of bone metastasis. N Engl J Med 2004;350:1655-64.

6. Yan M SS, Jiang ZF, et al. The clinical course of bone metastases of breast cancer. Zhong Guo Gu Zhong Liu Gu Bing 2003;2:221-4.

7. Working group for Guidelines of Chinese Society of Clinical Oncology. Chinese Soceity of Clinical Oncology (CSCO): Guidelines for Diagnosis and Treatment of Breast Cancer (2020). People's Medical Publishing House.

8. Chow E, Harris K, Fan G, et al. Palliative radiotherapy trials for bone metastases: a systematic review. J Clin Oncol 2007;25:1423-36.

9. British Association of Surgical Oncology Guidelines. The management of metastatic bone disease in the United Kingdom. The Breast Specialty Group of the British Association of Surgical Oncology. Eur J Surg Oncol 1999;25:3-23.

10. WHO. Medical need for opioid analgesics. Achieving balance in national opioids control policy: Guidelines for assessment. Geneva: WHO Publication. 2000:3-4.

11. Russell RG. Bisphosphonates: from bench to bedside. Ann
N Y Acad Sci 2006;1068:367-401.

12. Russell RG, Xia Z, Dunford JE, et al. Bisphosphonates: an update on mechanisms of action and how these relate to clinical efficacy. Ann N Y Acad Sci 2007;1117:209-57.

13. Thomas D, Henshaw R, Skubitz K, et al. Denosumab in patients with giant-cell tumour of bone: an open-label, phase 2 study. Lancet Oncol 2010;11:275-80.

14. Stopeck AT, Lipton A, Body JJ, et al. Denosumab compared with zoledronic acid for the treatment of bone metastases in patients with advanced breast cancer: a randomized, double-blind study. J Clin Oncol 2010;28:5132-9.

15. Cleeland CS, Body JJ, Stopeck A, et al. Pain outcomes in patients with advanced breast cancer and bone metastases: results from a randomized, double-blind study of denosumab and zoledronic acid. Cancer 2013;119:832-8.

16. Martin M, Bell R, Bourgeois H, et al. Bone-related complications and quality of life in advanced breast cancer: results from a randomized phase III trial of denosumab versus zoledronic acid. Clin Cancer Res 2012;18:4841-9.

17. Weitzman R, Sauter N, Eriksen EF, et al. Critical review: updated recommendations for the prevention, diagnosis, and treatment of osteonecrosis of the jaw in cancer patients--May 2006. Crit Rev Oncol Hematol 2007;62:148-52.

18. Jiang Z, Tang ET, Li C, et al. What is the relationship between bone turnover markers and skeletal-related events in patients with bone metastases from solid tumors and in patients with multiple myeloma? A systematic review and meta-regression analysis. Bone Rep 2020;12:100272.

19. Mjelstad A, Zakariasson G, Valachis A. Optimizing antiresorptive treatment in patients with bone metastases: time to initiation, switching strategies, and treatment duration. Support Care Cancer 2019;27:3859-67.

20. Brufsky AM, Harker WG, Beck JT, et al. Final 5-year results of Z-FAST trial: adjuvant zoledronic acid maintains bone mass in postmenopausal breast cancer patients receiving letrozole. Cancer 2012;118:1192-201.

21. Bundred NJ, Campbell ID, Davidson N, et al. Effective inhibition of aromatase inhibitor-associated bone loss by zoledronic acid in postmenopausal women with early breast cancer receiving adjuvant letrozole: ZO-FAST Study results. Cancer 2008;112:1001-10.

22. Llombart A, Frassoldati A, Paija O, et al. Immediate Administration of Zoledronic Acid Reduces Aromatase Inhibitor-Associated Bone Loss in Postmenopausal 
Women With Early Breast Cancer: 12-month analysis of the E-ZO-FAST trial. Clin Breast Cancer 2012;12:40-8.

23. Gnant M, Mlineritsch B, Schippinger W, et al. Endocrine therapy plus zoledronic acid in premenopausal breast cancer. N Engl J Med 2009;360:679-91.

doi: $10.21037 /$ tbcr-20-65

Cite this article as: Jiang $\mathrm{Z}$, Wang $\mathrm{H}$, Wang $\mathrm{S}$, Wang $\mathrm{S}$, Wang T, Wang X, Feng J, Liu J, Liu Q, Sun T, Yan M, Zhang Q, Chen J, Ouyang Q, Hu X, Geng C, Yuan P, Yin Y, Liao N, Pan Y. Chinese expert consensus statement on the clinical diagnosis and treatment of breast cancer bone metastasis and bone related disease. Transl Breast Cancer Res 2021;2:2.
24. Gnant M, Pfeiler G, Dubsky PC, et al. Adjuvant denosumab in breast cancer (ABCSG-18): a multicentre, randomised, double-blind, placebo-controlled trial. Lancet 2015;386:433-43.

(English Language Editor: J. Gray) 